\title{
Telomeric repeat-containing ribonucleic acid (TERRA) expression in patients with idiopathic pulmonary fibrosis
}

\author{
Manal M. El-Desoky ${ }^{1 *}$ D, Asem A. Hewidy², Ahmed M. Fouda ${ }^{2}$ and Fatma Azzahraa Hisham
}

\begin{abstract}
Background: Idiopathic pulmonary fibrosis (IPF) represents a chronic disease with a progressive course. It is characterized by excessive lung scarring that ultimately contributes to irreversible lung function reduction. Interestingly, a type of long non-coding RNA termed as telomeric repeat-containing RNA (TERRA) is linked to fibrosis pathophysiology, including IPF. In this study, the expression profile of TERRA was investigated in IPF patients on radiological diagnosis [unusual interstitial pattern (UIP) in high-resolution computed tomography (HRCT)] to evaluate whether it could be employed as a reliable diagnostic biomarker.

Results: TERRA expression level was significantly higher in IPF patients over healthy controls. The expression level was significantly inversely correlated with the percentage of forced vital capacity predicted (FVC\% predicted). By contrast, it was significantly directly correlated with HRCT reticular extent score.

Conclusion: TERRA expression is an essential biomarker in peripheral blood of IPF patients, providing a valuable non-invasive tool for IPF diagnosis. Moreover, TERRA expression is strongly correlated with UIP in HRCT reticular extent score.
\end{abstract}

Keywords: Gene expression, Idiopathic pulmonary fibrosis, Long noncoding ribonucleic acid, Telomeric repeatcontaining ribonucleic acid, Unusual interstitial pattern

\section{Background}

Idiopathic pulmonary fibrosis (IPF) is a specific fibrotic disease that has a chronic and progressive nature. This disorder has been associated with severe morbidity and poor prognostic rates [1]. Age is a significant risk factor contributing to the incidence of IPF; at the time of diagnosis, a major percentage of IPF patients are more than 60 years old. However, younger individuals are less affected by IPF [2]. It is estimated that IPF has a prevalence of 13 to 20 per 100,000 people worldwide. In the USA, approximately 100,000 persons are affected, and 30,000 to 40,000 new cases are diagnosed each year

\footnotetext{
* Correspondence: drmanal@live.com

${ }^{1}$ Department of Medical Biochemistry, Faculty of Medicine, Mansoura University, Mansoura, Egypt

Full list of author information is available at the end of the article
}

(https://medlineplus.gov/genetics/condition/idiopathicpulmonary-fibrosis/\#causes).

The hallmark radiological pattern of IPF is an unusual interstitial pattern (UIP). The distinguishing feature of UIP is a honeycombing sign which must be present to make a definite diagnosis of UIP on high-resolution computed tomography (HRCT). Subpleural with basal predominance and some upper lobe involvement are the typical sites for UIP distribution. In some cases, a relative uniform craniocaudal distribution of UIP may be present [3].

Long non-coding ribonucleic acids (lncRNAs) are non-coding transcripts with a length of more than 200 nucleotides. They are considered a novel class of epigenetic regulators that are attracting attention as they are involved in various biological processes [4].

\section{Springer Open}

(- The Author(s). 2021 Open Access This article is licensed under a Creative Commons Attribution 4.0 International License, which permits use, sharing, adaptation, distribution and reproduction in any medium or format, as long as you give appropriate credit to the original author(s) and the source, provide a link to the Creative Commons licence, and indicate if changes were made. The images or other third party material in this article are included in the article's Creative Commons licence, unless indicated otherwise in a credit line to the material. If material is not included in the article's Creative Commons licence and your intended use is not permitted by statutory regulation or exceeds the permitted use, you will need to obtain permission directly from the copyright holder. To view a copy of this licence, visit http://creativecommons.org/licenses/by/4.0/. 
There is a close relationship between the expression of lncRNAs and various metabolic diseases, autoimmune diseases, hereditary diseases, and tumors. Moreover, they are recognized as fibrosis functional regulators. These RNAs have a secondary role in several diseases and a primary fibrosis pathology. Also, several lncRNAs are involved in the development of epithelial-mesenchymal transition of alveolar epithelial cells, which is the main pathogenic mechanism of idiopathic fibrosis [5].

TERRA is one type of the lncRNAs that is transcribed from sub-telomeric sequences towards the ends of the chromosome by RNA polymerase II. TERRA transcripts carry telomeric repetitions in their sequences and are involved in the maintenance and control of telomeric homeostasis [6]. The chromosome ends of telomeres deteriorate in the absence of such regulatory mechanisms; as a result, DNA damage will be promoted due to the dysfunctional telomeres. Genome instability, cellular senescence, and/or apoptosis are consequences of this deterioration [7]. TERRA and other types of lncRNA overexpression have some evidence to be induced in highly proliferative tissues, especially IPF.

Previous studies were conducted depending on histopathological characteristics in which many patients were not fit for a bronchoscopy. In IPF patients, we assessed TERRA expression depending on radiological diagnosis (UIP pattern in HRCT) and determine whether it can be applied as a diagnostic biomarker in our locality instead of invasive procedures.

\section{Methods}

\section{Patients}

This was a case-control study that enrolled 30 IPF patients and 30 healthy controls of the same age and sex. IPF was diagnosed according to the American Thoracic Society/European Respiratory Society consensus criteria [3] which define the clinical, laboratory, and radiographic characteristics. There was an obtained written informed consent from the study participants. The institutional Ethical Committee approved this study with approval number 524 in May 2019.

\section{Inclusion criteria}

Inclusion criteria for this study were (1) age between 40 and 75 years as IPF is a disease of old patient and (2) newly diagnosed IPF based on UIP pattern in HRCT of the chest [3].

\section{Exclusion criteria}

The exclusion criteria were as follows: (1) previous steroid therapy or other immunosuppressive drugs, (2) atypical pattern of UIP on HRCT of the chest, and (3) patients with other known causes of interstitial lung disease (ILD).

\section{Methods}

All the patients were subjected to the following:

1) Full medical history including occupational, environmental, drug history, and chest symptoms suggestive of IPF (e.g., dry cough and dyspnea).

2) General and physical clinical examination.

3) Autoimmune profile to exclude autoimmune causes of ILD.

4) Radiological investigation: chest X-ray and HRCT of the chest in which a score was done for HRCT chest pattern of IPF (i.e., honeycombing, reticular pattern, and ground glass score) [8].

5) Extraction of total RNA and synthesis of cDNA.

Five milliliters of blood was obtained from each participant on ethylenediaminetetraacetic acid (EDTA) collection tubes. According to the manufacturer's instructions, peripheral mononuclear blood cell (PBMC) isolation was performed using Histopaque-1077 (SigmaAldrich) Ficoll density-gradient centrifugation.

Total RNAs were isolated from PBMCs using TRIzol reagent (Zymo Research, Irvine, CA). The concentration and purity of RNA have been evaluated by RNA NanoDrop2000 (Thermo Fischer Scientific, Waltham, MA). The A260/A280 and A260/A230 ratios were > 1.8 and > 1.7 , respectively. A temperature of $-80{ }^{\circ} \mathrm{C}$ was used to keep the total RNA before use. SensiFAST cDNA Synthesis kit (Bioline, Memphis, TN) has been used for reverse transcription of $1 \mu \mathrm{g}$ total RNA to cDNA with a final volume of $20 \mathrm{ml}$ reactions $(1 \mu \mathrm{l}$ cDNA, $4 \mu \mathrm{l} 5 \mathrm{x}$ TransAmp Buffer, $1 \mu \mathrm{l}$ reverse transcriptase, and $13 \mu \mathrm{l}$ DNase/RNase-free water).

\section{6) Real-time quantitative PCR}

HERA PLUS SYBR Green qPCR Kit (Willowfort, Birmingham, UK) was used to evaluate the expression levels of the IncRNA TERRA. PCR reaction $(20 \mu \mathrm{l})$ of $10 \mu \mathrm{l}$ SYBR Green Mix (2x), $1 \mu \mathrm{l}$ of cDNA, $1 \mu \mathrm{l}$ of each primer, and 7 $\mu \mathrm{l}$ nuclease-free $\mathrm{H}_{2} \mathrm{O}$ was performed using an Applied Biosystems 7500 Real-Time PCR System (Applied Biosystems, Waltham, MA). The real-time PCR thermal conditions were as follows: initial denaturation at $95^{\circ} \mathrm{C}$ for $10 \mathrm{~min}$, followed by 40 cycles of denaturation at $95^{\circ} \mathrm{C}$ for $15 \mathrm{~s}$, annealing at $60^{\circ} \mathrm{C}$ for $60 \mathrm{~s}$, and extension at $72{ }^{\circ} \mathrm{C}$ for $60 \mathrm{~s}$. Fold-change was calculated using the comparative threshold cycle $\left(2^{-\Delta \Delta C t}\right)$ [9]. This analysis has been confirmed by agarose electrophoresis (Fig. 1). The primers were provided as follows: TERRA- forward was 5-', CCTAACCCTC ACCCTTCTAAC-3 and -reverse was 5-', TACCTCGCTT TGGGACAAC-3 and GAPDH Forward Primer: 5'-, GTCAAGGCTGAGAACGGGAA-3" and GAPDH Reverse Primer: 5' -', AAATGAGCCCCAGCCTTCTC-3". 


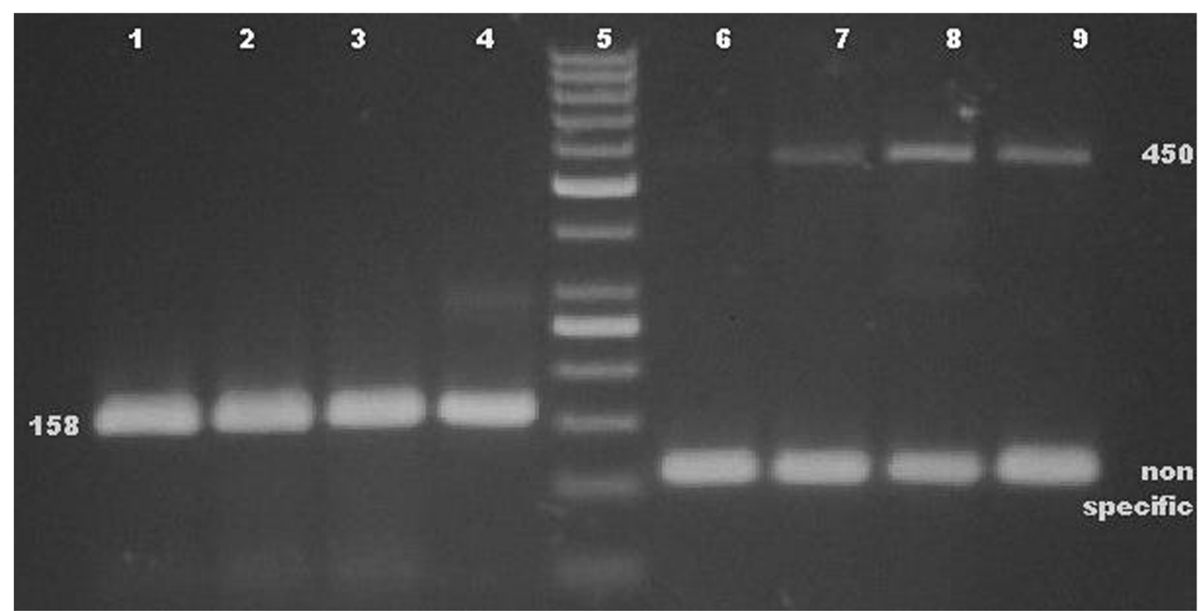

Fig. 1 RT-PCR product of TERRA gene expression and expression of GAPDH (control housekeeping gene). Lanes 1, 2, 3, and 4 represent PCR product of $158 \mathrm{bp}$ for GAPDH; lane 5 represents the $50 \mathrm{bp}$ ladder; lanes 6, 7, 8, and 9 represent PCR product of 450 bp for TERRA

\section{Statistical analysis}

Software for SPSS (version 24.; IBM, Inc., Chicago, IL) was used to analyze the obtained data. First, the Shapiro-Wilk normality test was used to analyze the normality of data. Continuous variables including parametric data were presented as means \pm SD (standard deviation), and nonparametric data were presented by median (minimum-maximum). Student's $t$-test was used for parametric data while Mann-Whitney test was used for nonparametric data. Continuous data was correlated by Spearman correlation. ROC curve was used to test sensitivity and specificity at different cutoff points. $p$ value $<0.05$ was considered as statistically significant.

\section{Results}

This study included 30 IPF patients and 30 healthy controls with a mean age of $63.40 \pm 5.58$ and $62.90 \pm 9$ years, respectively. There were no statistically significant differences between the two groups regarding age, sex, occupation, or smoking status. There was a statistically significant increase in symptoms suggestive of IPF (e.g., dyspnea $\leq 0.001$ and dry cough $\leq 0.001$ ) in case group relative to healthy controls (Table 1). In patient group, only one wheezy patient and two patients with diffuse chest pain.

The results revealed significant decrease in the FVC\% predicted $(p \leq 0.001)$ and diffusing capacity of the lung for carbon monoxide (DLCO\%) $(p \leq 0.001)$ in IPF patients compared to controls. On the other hand, forced expiratory volume (FEV1 \%predicted) was lower in patient group than healthy control but it is not statistically significant $(p=0.078)$.

Regarding the HRCT pattern, there was a statistically significant increase in reticular $(p \leq 0.001)$, honeycombing $(p \leq 0.001)$, and ground glass opacity (GGO) extent score $(p=0.033)$ in IPF patients relative to control group. TERRA relative expression was significantly higher in case group compared to controls $(p \leq 0.001)$ (Table 2 and Fig. 2).

TERRA expression level was significantly positively correlated with the patient age $(p=0.0001)$, duration of symptoms $(p \leq 0.001)$, and HRCT reticular extent score $(p=0.008)$. By contrast, it was negatively correlated with FVC $\%$ predicted $(p=0.034)$ (Figs. 3, 4, 5 and 6, Table 3).

The results showed no significant correlation between TERRA expression level with HRCT honeycombing extent score, GGOs extent score, and clinical symptoms (Table 3).

ROC curve analysis was done between TERRA expression levels in patients and controls with an area under the curve of $0.951\left(P^{<} 0.0001\right)$. At a cutoff point of 1.13 , the sensitivity was $93.3 \%$, the specificity was $86.7 \%$, and the total accuracy was $90 \%$ (Fig. 7).

\section{Discussion}

IPF is a disorder of progressive course with fatal complications. The etiology and pathogenesis of this disease are still unclear [8]. The development and progression of IPF have been strongly related to activation of lung fibroblast and epithelial cell, as well as fibrotic and inflammatory mediators secretion [10].

This study revealed that the most prevalent clinical complaints, such as dry cough and marked dyspnea, were significantly increased in IPF patients. Regarding pulmonary function tests, the patients in the present study are distinguished by restrictive lung disease patterns (FVC\% of predicted and DLCO). These findings were expected because of the nature of interstitial lung diseases. 
Table 1 Comparison between IPF patients and control groups regarding demographic and clinical data

\begin{tabular}{|c|c|c|c|c|}
\hline Variables & IPF patients group ( $\left.n \_30\right)$ & Control group (n_30) & Test of significance & $P$-value \\
\hline \multicolumn{5}{|l|}{ Age (years) } \\
\hline Mean \pm SD & $63.40 \pm 5.58$ & $62.90 \pm 9.91$ & $t=0.241$ & 0.811 \\
\hline \multicolumn{5}{|l|}{ Sex } \\
\hline Male & $23-(76.7 \%)$ & $23-(76.7 \%)$ & $x^{2}=0.00$ & 1.00 \\
\hline Female & 7-(23.3\%) & 7-(23.3\%) & & \\
\hline \multicolumn{5}{|l|}{ Smoking } \\
\hline Smoker & $21(70 \%)$ & $14(46.7 \%)$ & $x^{2}=3.36$ & 0.067 \\
\hline Nonsmoker & $9(30 \%)$ & $16(53.3 \%)$ & & \\
\hline \multicolumn{5}{|l|}{ Occupation } \\
\hline Non-worker & $13(43.3 \%)$ & $9(30 \%)$ & $M C$ & 0.603 \\
\hline Manual worker & $14(46.7 \%)$ & $18(60 \%)$ & & \\
\hline Professional worker & $3(10 \%)$ & $3(10 \%)$ & & \\
\hline \multicolumn{5}{|l|}{ Dyspnea grade } \\
\hline 0 & $1(3.3 \%)$ & 27 (90\%) & $M C$ & $\leq 0.001^{*}$ \\
\hline 1 & $4(13.3 \%)$ & $3(10 \%)$ & & \\
\hline 2 & $12(40.0 \%)$ & $0(0 \%)$ & & \\
\hline 3 & $11(36.7 \%)$ & $0(0 \%)$ & & \\
\hline 4 & $2(6.7 \%)$ & $0(0 \%)$ & & \\
\hline \multicolumn{5}{|l|}{ Cough grade } \\
\hline 0 & $1(3.3 \%)$ & $24(80 \%)$ & $M C$ & $\leq 0.001^{*}$ \\
\hline 1 & $13(43.3 \%)$ & $6(20 \%)$ & & \\
\hline 2 & 16 (53.3\%) & $0(0 \%)$ & & \\
\hline
\end{tabular}

$t$ Student $t$-test, $X^{2}$ Chi square test, $M C$ Monte Carlo test

*Significant $p<0.05$

Non-coding RNAs, including lncRNAs with a length of over 200 nucleotides, are the major transcription products in mammalian cells. Although several studies have started to clarify lncRNA function in human diseases, lncRNA expression profiles, as well as roles in IPF, remain unclear.

Herein, a PCR-based method was utilized to evaluate the TERRA expression level in peripheral blood of IPF patients. Although there have not been many studies conducted on TERRA expression in IPF, we found that increased TERRA expression is significantly related to an increase in IPF incidence. This is consistent with the findings of Gao et al. [7] who demonstrated that an increase in TERRA expression levels of IPF patients, along with its association with dysfunction of telomeres caused by oxidative stress, contributes to IPF pathogenesis.

Table 2 Comparison between IPF patients and control groups regarding pulmonary function tests, UIP pattern in HRCT, and relative expression of TERRA mRNA

\begin{tabular}{|c|c|c|c|c|}
\hline Variables & IPF patient group ( $\left.n \_30\right)$ & Control group ( $n \_30$ ) & Test of significance & $P$-value \\
\hline FEV1 \%predicted & $79.91 \pm 10.13$ & $84.71 \pm 10.60$ & $t=1.79$ & 0.078 \\
\hline FVC\% predicted & $72.87 \pm 9.73$ & $94.42 \pm 10.41$ & $t=8.27$ & $\leq 0.001^{*}$ \\
\hline \multicolumn{5}{|l|}{ DLCO\% } \\
\hline Median (min-max) & $55.0(26-89)$ & $94.0(70-110)$ & $Z=9.75$ & $\leq 0.001^{*}$ \\
\hline HRCT reticular extent score & $2.00(1.00-4.00)$ & $0.00(0.00-1.00)$ & $Z=10.66$ & $\leq 0.001^{*}$ \\
\hline HRCT honeycombing extent score & $2.00(0.00-4.00)$ & $0.00(0.00-1.00)$ & $Z=8.94$ & $\leq 0.001^{*}$ \\
\hline HRCT GGOs extent score & $0.00(0.00-2.00)$ & $0.00(0.00-1.00)$ & $Z=2.13$ & $0.033^{*}$ \\
\hline Relative expression & $1.99 \pm 0.63$ & $0.97 \pm 0.12$ & $t=8.68$ & $\leq 0.001^{*}$ \\
\hline
\end{tabular}

$t$ Student $t$-test, $Z$ Mann-Whitney test

*Significant $p<0.05$ 


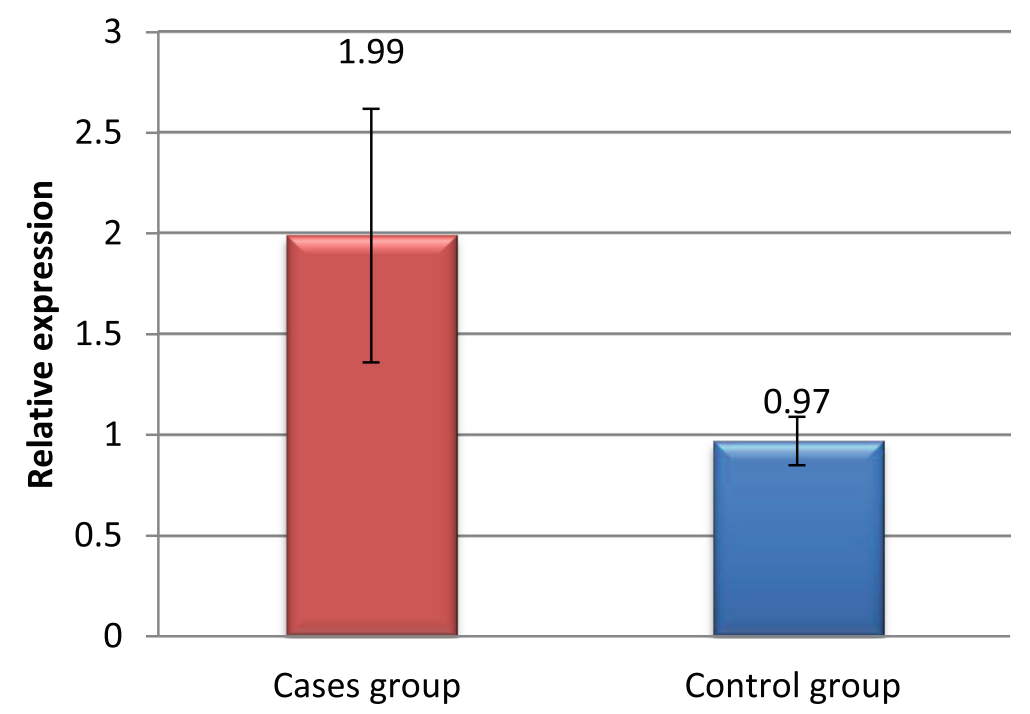

Fig. 2 TERRA mRNA expression in the patient with idiopathic pulmonary fibrosis with healthy control. Relative expression $=1.99 \pm 0.63(P$ $\leq 0.001$ )

Cao et al. [11] identified a large number of lncRNAs with altered expression in rat lungs using experimental fibrosis. They showed differences in the RNA component of mitochondrial RNA processing endoribonuclease expression in addition to telomere enzyme RNA component (Terc) in a bleomycin-induced fibrotic murine model. This was the first piece of evidence that suggests participation of lncRNAs in pathogenesis of pulmonary fibrosis. Moreover, pulmonary fibrosis can be induced by destructing CCAAT box in lncRNA Terc promoter [12]. Besides, Liu et al. [13] demonstrated that lncRNA telomerase reverse transcriptase (Tert) inactivation reduces the pulmonary fibrosis severity in mice exposed to conditional knockout.

Much evidence proposed that lncRNAs have a role in cell proliferation. The residential lung fibroblast

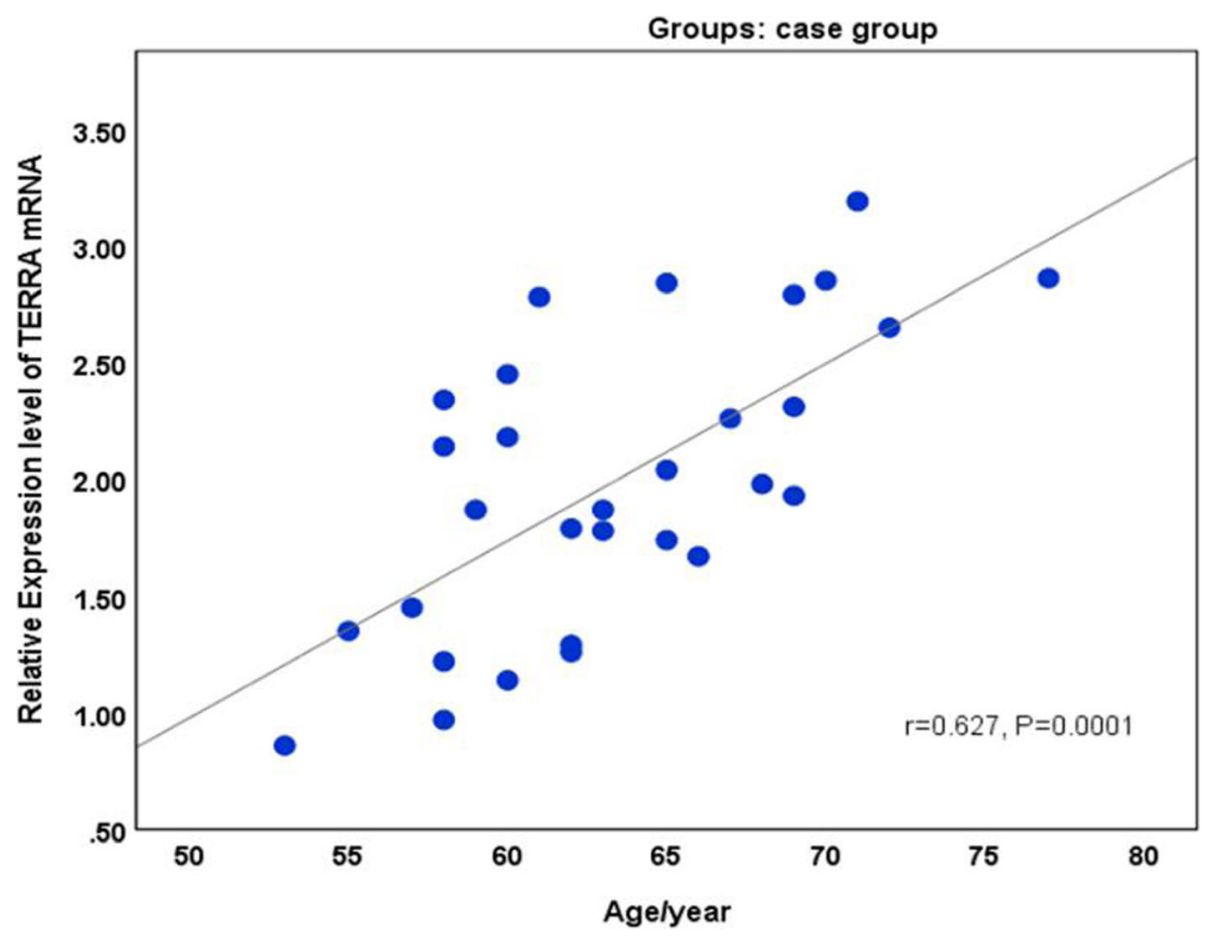

Fig. 3 Scatter diagram showing that both relative expression and age have a positive correlation 


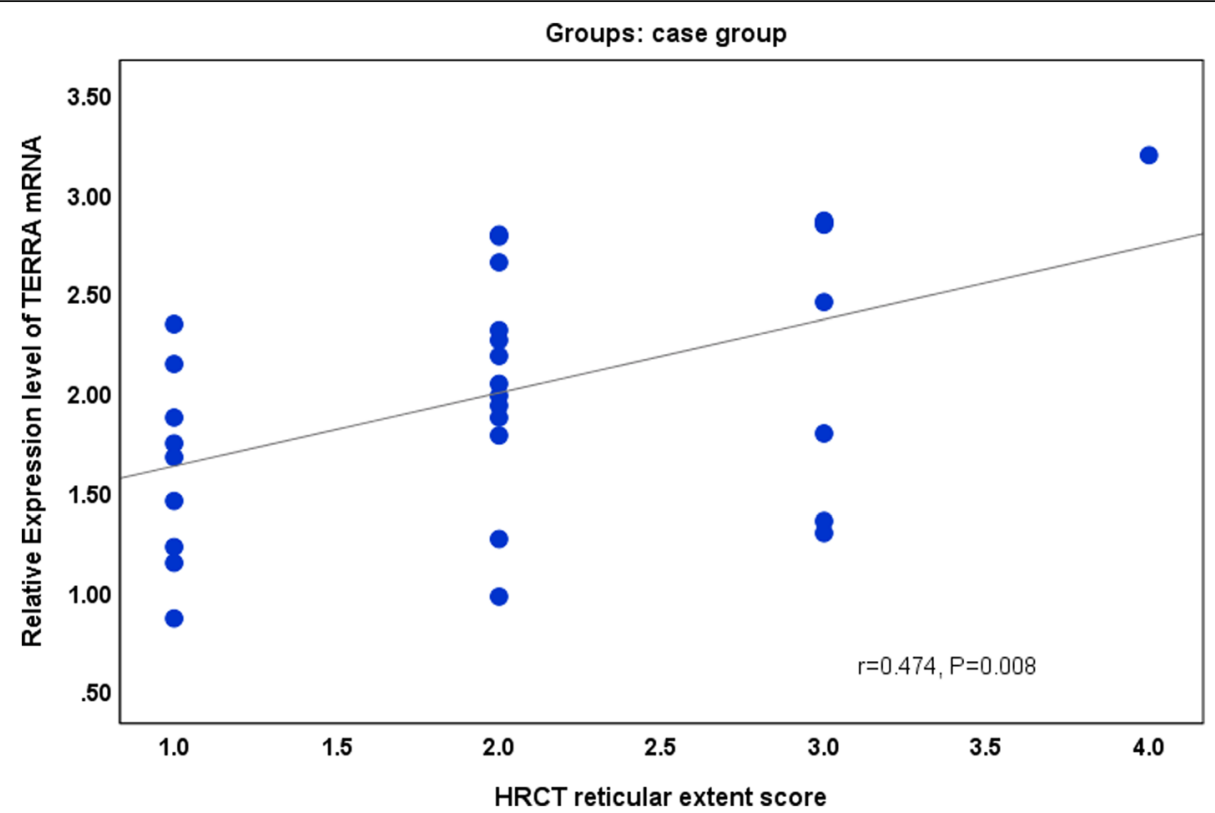

Fig. 4 Scatter diagram showing that both relative expression and the high-resolution computed tomography reticular extent score have a positive correlation

proliferation represents one of the leading providers that increase IPF fibroblastic foci [14]. Certain lncRNAs, namely LINC00960 and LINC01140, were suggested to be upregulated, and LINC01140 knockdown but not LINC00960 leads to stimulation of inflammatory response fibroblasts in IPF. As a result, this demonstrated the necessity of lncRNAs as both proliferation and inflammation regulators in IPF [15]. Besides, Dai et al. [16] found that MALAT1 in rat models was activated by the inflammatory response pathway caused by lipopolysaccharide and promoted lung injury progression.

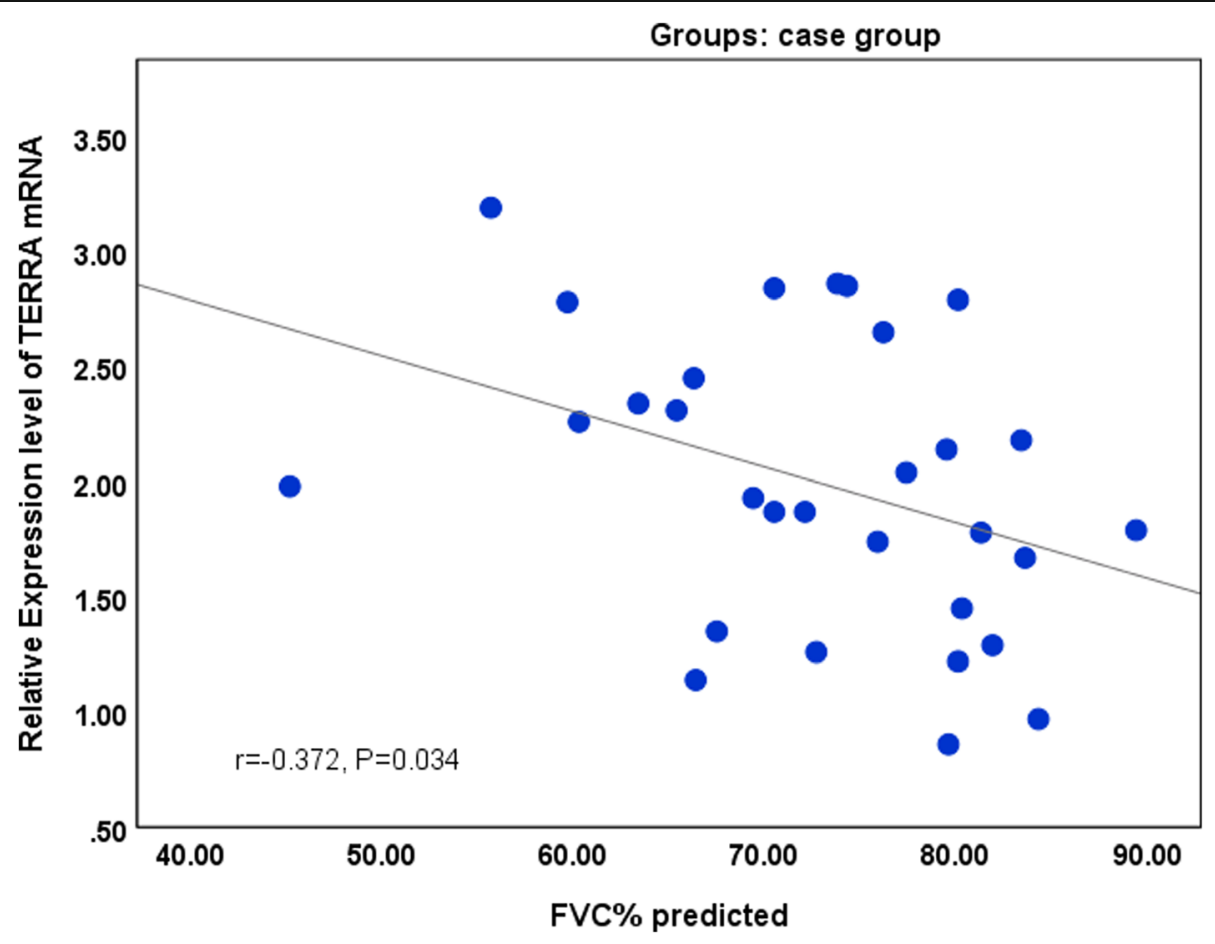

Fig. 5 Scatter diagram showing that both relative expression and duration of symptoms have a positive correlation 


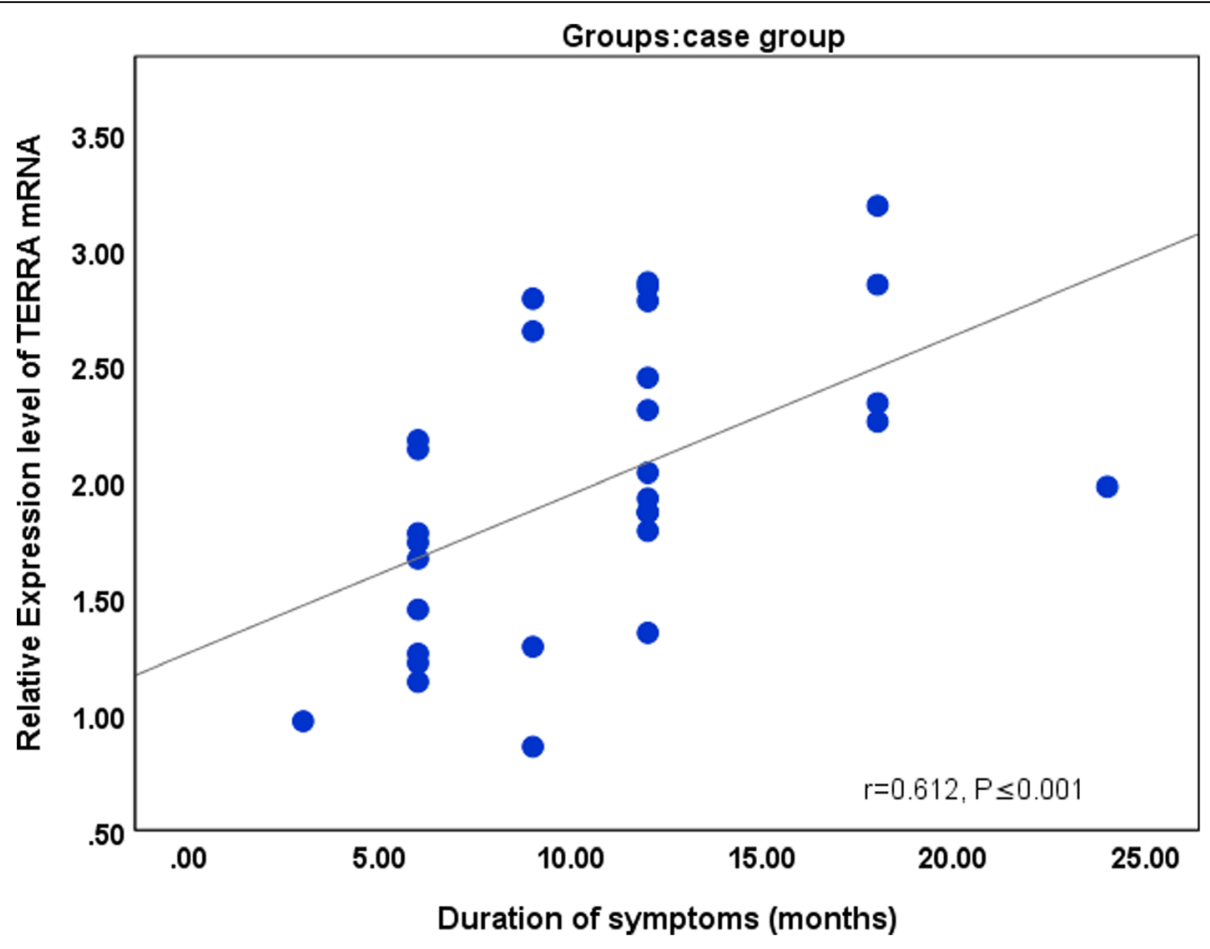

Fig. 6 Scatter diagram showing that both relative expression and predicted forced vital capacity to have a negative correlation

Numerous researches indicate different functions of lncRNAs like acting as miRNA sponges, or decoys, holding a role as competitive endogenous RNAs for microRNAs, and having importance in physiological as well as pathological events. Nevertheless, expression profiles, as well as IncRNA roles in IPF, remain unclear [14].

In the case of patients that have newly identified interstitial lung diseases (ILD) and possess an HRCT scan pattern of a UIP, they were strongly recommended against conducting surgical lung biopsy, transbronchial lung biopsy, in addition to lung cryobiopsy. Besides, a

Table 3 Correlation between TERRA expression and different clinical and radiological characteristic

\begin{tabular}{lll}
\hline Variables & \multicolumn{2}{l}{ Relative expression } \\
\cline { 2 - 3 } & $\boldsymbol{r}$ & $\boldsymbol{p}$ \\
\hline HRCT reticular extent score & 0.474 & $0.008^{*}$ \\
HRCT honeycombing extent score & 0.213 & 0.258 \\
HRCT GGOs extent score & 0.094 & 0.623 \\
Age & 0.627 & $0.0001^{*}$ \\
Duration of symptoms & 0.612 & $\leq 0.001^{*}$ \\
Dyspnea grade & -0.081 & 0.669 \\
Cough grade & 0.154 & 0.418 \\
FEV1 \%predicted & -0.183 & 0.332 \\
FVC\% predicted & -0.372 & $0.034^{*}$ \\
DLCO\% & -0.355 & 0.051 \\
\hline
\end{tabular}

*Significant $p<0.05$ conditional recommendation was made to conduct BAL after excluding other existing ILD causes (like domestic and occupational environmental exposures, connective tissue diseases, and drug toxicity) [3].

Our study showed a predominance of reticulation and honeycombing in HRCT equivalent to that observed in a study by Kono et al. [8] in which HRCT score interobserver agreement was good, and the honeycombing and reticulation extent scores were significantly higher in IPF than in non-IPF idiopathic interstitial pneumonia.

The results indicated a significant positive correlation between patient's age and TERRA expression levels. In addition, a progressive shortening of telomere length is commonly associated with cellular senescence. The IncRNA TERRA suppresses telomere elongation and modulates telomeric heterochromatin by linking with multiple telomeric proteins: repeat factor telomere (TRF1, TRF2), origin-recognition complex (ORC), and the H3K9me3 factor [17].

Hao et al. [18] demonstrated that patients with IPF demonstrated a remarkable increase of IncRNA AP003419.16 expression and its adjacent gene, RPS6KB2, and this may lead to increased risk of agingassociated IPF. Previous data suggested that lncRNAs are involved in regulating aging-associated disease [19] and aging pathways [20].

In this study, TERRA expression levels were inversely correlated with $\mathrm{FVC} \%$ of predicted and directly correlated with HRCT reticular extent score. Lung biopsy is 


\section{ROC Curve}

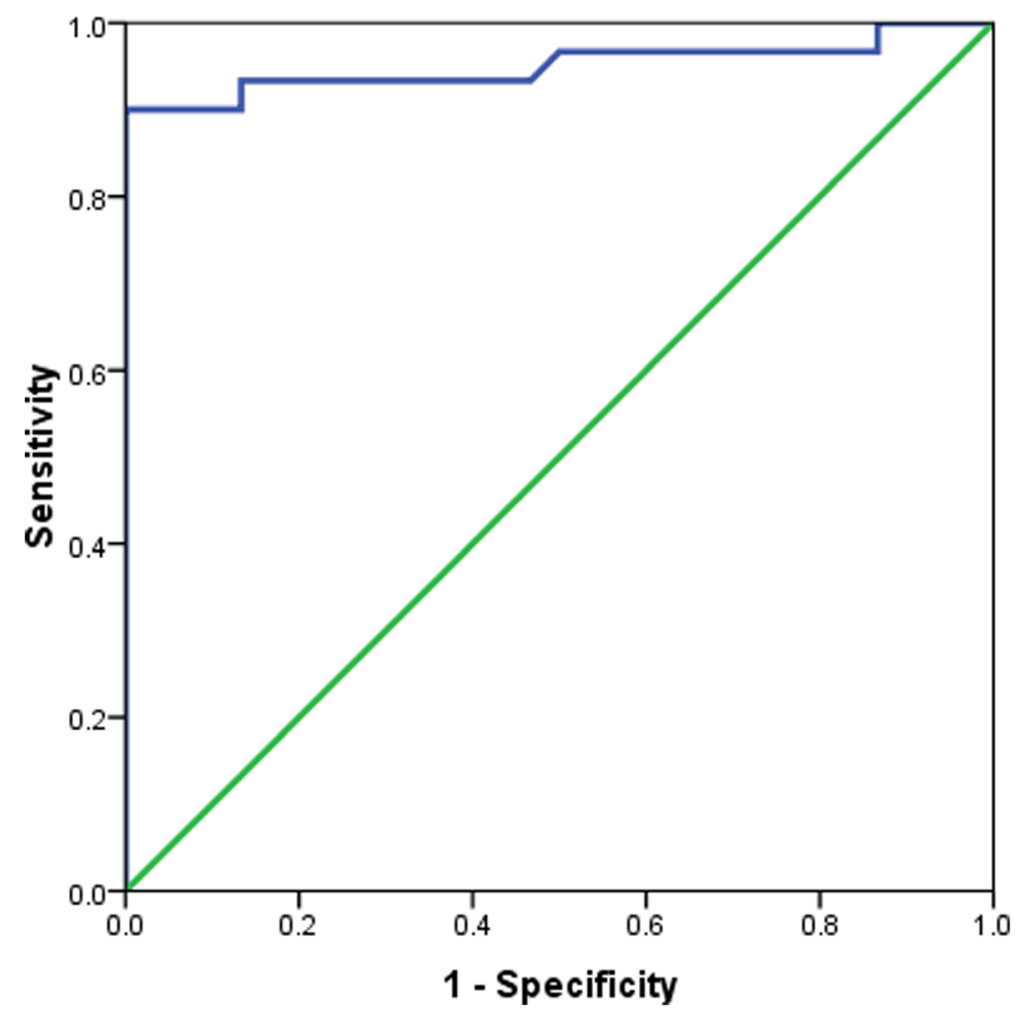

Fig. 7 The area under the receiver operating characteristics curve of telomeric repeat-containing RNA (TERRA) in idiopathic pulmonary fibrosis patients and controls

considered important as the only strategy that can give an accurate diagnosis for IPF. Furthermore, a biopsy of the lungs is an invasive procedure resulting in increased morbidity as well as mortality risks. TERRA research remains at an early stage, and the emphasis on TERRA research to find suitable therapies will continue to evolve in the future.

\section{Conclusion}

TERRA expression is an essential biomarker in peripheral blood of IPF patients, providing a valuable noninvasive tool for IPF diagnosis. Moreover, TERRA expression is strongly correlated with UIP in HRCT reticular extent score.

\section{Abbreviations}

HRCT: High-resolution computed tomography; ILD: Interstitial lung diseases; IPF: Idiopathic pulmonary fibrosis; IncRNA: Long non-coding RNA;

ORC: Origin-recognition complex; TERRA: Telomeric repeat-containing RNA;

TRF: Repeat factor telomere; UIP: Unusual interstitial pattern
Authors' contributions

$\mathrm{ME}$ and $\mathrm{FH}$ designed the research and performed the experiments. $\mathrm{AH}$ and AF recruited the patients and processed the samples. All authors analyzed the data, shared in writing the manuscript, and read and approved the final version of this manuscript

\section{Funding}

No funding was received.

\section{Availability of data and materials}

The datasets used and analyzed during the current study are available from the corresponding author on reasonable request.

\section{Ethics approval and consent to participate}

Research involving human subjects complied with all relevant national regulations and institutional policies and is in accordance with the tenets of the Helsinki Declaration [as revised in 2013], and has been approved by the Ethical Committee, Faculty of Medicine, Mansoura University, Egypt (R/ 19.05.524). Informed written consent was obtained from all individuals included in this study.

Consent for publication

Not applicable.

\section{Competing interests}

The authors declare that they have no competing interests. 


\section{Author details}

'Department of Medical Biochemistry, Faculty of Medicine, Mansoura University, Mansoura, Egypt. ${ }^{2}$ Department of Chest Medicine, Faculty of Medicine, Mansoura University, Mansoura, Egypt.

Received: 28 September 2020 Accepted: 2 February 2021

Published online: 19 March 2021

\section{References}

1. Sgalla G, lovene B, Calvello M, Ori M, Varone F, Richeldi L (2018) Idiopathic pulmonary fibrosis: pathogenesis and management. Respir Res 19:32

2. Raghu G, Weycker D, Edelsberg J, Bradford WZ, Oster G (2006) Incidence and prevalence of idiopathic pulmonary fibrosis. Am J Respir Critical Care Med 174:810-816

3. Raghu G, Remy-Jardin M, Myers JL, Richeldi L, Ryerson CJ, Lederer DJ et al (2018) Diagnosis of idiopathic pulmonary fibrosis. An official ATS/ERS/JRS/ ALAT clinical practice guideline. Am J Respir Critical Care Med 198:44-68

4. Sakamoto N, Honma R, Sekino Y, Goto K, Sentani K, Ishikawa A et al (2017) Noncoding RNAs are promising targets for stem cell-based cancer therapy. Non-coding RNA Res 2:83-87

5. Zhang Y, Luo G, Zhang Y, Zhang M, Zhou J, Gao W et al (2018) Critical effects of long noncoding RNA on fibrosis diseases. Exp Mol Med 50:e428

6. Azzalin CM, Lingner J (2015) Telomere functions grounding on TERRA firma. Trends Cell Biol 25:29-36

7. Gao Y, Zhang J, Liu Y, Zhang S, Wang Y, Liu B et al (2017) Regulation of TERRA on telomeric and mitochondrial functions in IPF pathogenesis. BMC Pulm Med 17(1):1-15

8. Kono M, Nakamura Y, Suda T, Kato M, Kaida Y, Hashimoto D et al (2011) Plasma CCN2 (connective tissue growth factor; CTGF) is a potential biomarker in idiopathic pulmonary fibrosis (IPF). Clin Chim Acta 412:2211-2215

9. Schmittgen TD, Livak KJ (2008) Analyzing real-time PCR data by the comparative C(T) method. Nat Protoc 3:1101-1108

10. Hadjicharalambous MR, Lindsay MA (2020) Idiopathic pulmonary fibrosis: pathogenesis and the emerging role of long noncoding RNAs. Int J Mol Sci 21:524-543

11. Cao G, Zhang J, Wang M, Song X, Liu W, Mao C et al (2013) Differential expression of long non-coding RNAs in bleomycin-induced lung fibrosis. Int J Mol Med 32:355-364

12. Aalbers AM, Kajigaya S, van den Heuvel-Eibrink MM, van der Velden VH, Calado RT, Young NS (2012) Human telomere disease due to disruption of the CCAAT box of the TERC promoter. Blood 119:3060-3063

13. Liu T, Yu H, Ding L, Wu Z, Gonzalez De Los Santos F, Liu J, Ullenbruch M et al (2015) Conditional knockout of telomerase reverse transcriptase in mesenchymal cells impairs mouse pulmonary fibrosis. PLoS One 10:e142547

14. Huang C, Yang Y, Liu L (2015) Interaction of long noncoding RNAs and microRNAs in the pathogenesis of idiopathic pulmonary fibrosis. Physiol Genomics 47:463-469

15. Hadjicharalambous MR, Roux BT, Csomor E, Feghali-Bostwick CA, Murray LA Clarke D et al (2019) Long intergenic non- coding RNAs regulate human lung fibroblast function: implications for idiopathic pulmonary fibrosis. Sci Rep 9(1):6020

16. Dai L, Zhang G, Cheng Z, Wang X, Jia L, Jing X et al (2018) Knockdown of InCRNA MALAT1 contributes to the suppression of inflammatory responses by up-regulating miR-146a in LPS-induced acute lung injury. Connect Tissue Res 59(6):581-592

17. Deng Z, Norseen J, Wiedmer A, Riethman H, Lieberman PM (2009) TERRA RNA binding to TRF2 facilitates heterochromatin formation and ORC recruitment at telomeres. Mol Cell 35:403-413

18. Hao X, Du Y, Qian L, Li D, Liu X (2017) Upregulation of long noncoding RNA AP003419.16 predicts high risk of aging-associated idiopathic pulmonary fibrosis. Mol Med Rep 16:8085-8091

19. Li X, Wu Z, Fu X, Han W (2014) IncRNAs: insights into their function and mechanics in underlying disorders. Mutat Res Rev Mutat Res 762:1-21

20. Yang D, Lian T, Tu J, Gaur U, Mao X, Fan X et al (2016) LncRNA mediated regulation of aging pathways in Drosophila melanogaster during dietary restriction. Aging (Albany NY) 8:2182-2203

\section{Publisher's Note}

Springer Nature remains neutral with regard to jurisdictional claims in published maps and institutional affiliations.

\section{Submit your manuscript to a SpringerOpen ${ }^{\circ}$ journal and benefit from:}

- Convenient online submission

- Rigorous peer review

- Open access: articles freely available online

- High visibility within the field

- Retaining the copyright to your article

Submit your next manuscript at $\boldsymbol{\nabla}$ springeropen.com 